\title{
Cerebral bypass surgery for skull base lesions: technical notes incorporating lessons learned over two decades
}

\author{
Ketan R. Bulsara, M.D., ${ }^{1}$ Toral Patel, M.D., ${ }^{1}$ ANd Takanori Fukushima, M.D., \\ D.S.M. ${ }^{2,3}$ \\ ${ }^{1}$ Department of Neurosurgery, Yale University School of Medicine, New Haven, Connecticut; \\ ${ }^{2}$ Carolina Neuroscience Institute, Raleigh; and ${ }^{3}$ Division of Neurosurgery, \\ Duke University School of Medicine, Durham, North Carolina
}

\begin{abstract}
Object. Despite advancements in endovascular neurosurgery, there remains an important role for cerebral bypass surgery in the treatment of skull base lesions. The authors describe their replacement cerebral bypass surgery techniques incorporating lessons learned over 2 decades.

Methods. The authors performed a retrospective review of cerebral bypass surgery performed by the senior author for skull base lesions between 1986 and 2006. One hundred patients had adjunct bypass surgery for skull base lesions.

Results. The bypass surgeries performed are conceptually divided into Skull Base Bypass I, II, and III. The majority of lesions requiring bypass surgery were giant cavernous carotid artery aneurysms or skull base meningiomas. There were no deaths in this case series. There was a $7 \%$ morbidity rate.

Conclusions. The ability to perform this surgery is an important adjunct in the armamentarium of skull base/cerebrovascular neurosurgeons. (DOI: 10.3171/FOC/2008/24/2/E11)
\end{abstract}

KEY WORDS • cerebral bypass • revascularization • skull base surgery

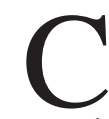
EREBRAL bypass surgery indications have undergone significant modifications. Despite the revolution in endovascular neurosurgery, cerebral bypass surgery remains an essential component in the management of some skull base lesions. In this paper, we describe our cerebral bypass surgery techniques, incorporating lessons learned over 2 decades.

\section{Clinical Materials and Methods}

Between 1986 and 2006, the senior author treated 100 skull base lesions with adjunctive bypass surgery. Saphenous vein grafts were used in all cases. During the bypass procedures, moderate intraoperative anticoagulation therapy (heparin 3000-5000 units) was utilized. Postoperative CT studies were obtained approximately 6 hours after the procedure. If there was no evidence of any hemorrhage, oral or suppository aspirin therapy was initiated. In patients who were slow to mobilize, deep vein thrombosis prophylaxis with lovenox was initiated 24 hours after the procedure.

\section{Results}

The majority of lesions treated were giant cavernous CA

Abbreviations used in this paper: $\mathrm{CA}=$ carotid artery; $\mathrm{CT}=$ computed tomography; ICA = internal carotid artery. aneurysms or skull base meningiomas. Other lesions included malignant tumors involving the skull base, highcervical ICA aneurysms, carotid body tumors, glomus jugulare tumors, and complex intracranial aneurysms. There were no deaths in this case series. The morbidity rate was $7 \%$. The major morbidity was some degree of visual loss in the management of giant cavernous CA aneurysms, occurring in $8 \%$ of these patients. There was 1 acute occlusion of a petrous segment-siphon bypass with resultant hemiplegia. Among the high-cervical segment-petrous segment bypasses there was 1 late occlusion at 6 months. It did not result in any deficits.

\section{Discussion}

Even though CA sacrifice without bypass is an option, we try to preserve the CA whenever possible. Preoperative balloon test occlusion was performed for prognostic purposes in the patients in this case series.

Conceptually, we have divided the skull base bypasses into 3 groups: Skull Base Bypass I (petrous segmentsiphon saphenous vein graft; Fig. 1); Skull Base Bypass II (high-cervical segment-petrous segment bypass; Fig. 2); Skull Base Bypass III (high-cervical segment-siphon or $\mathbf{M}_{2}$ saphenous vein graft; Fig. 3). The details of these procedures as outlined in the senior author's manual ${ }^{1}$ are presented below. 


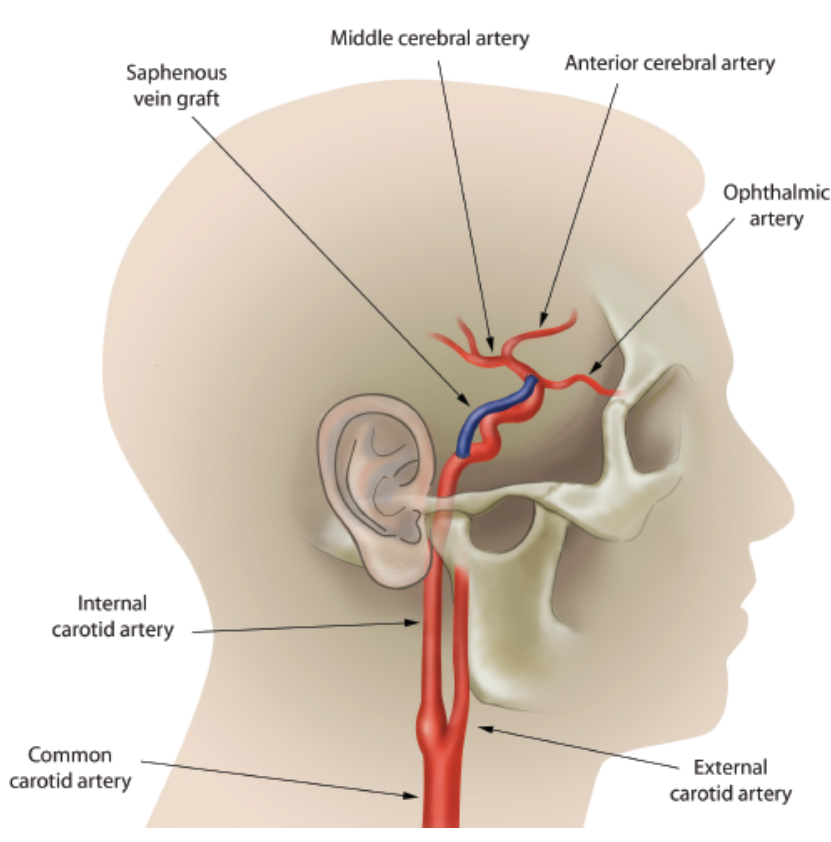

FIG. 1. Schematic illustration of Skull Base Bypass I.

\section{The Skull Base Bypass I}

The petrous segment-siphon saphenous vein bypass (Skull Base Bypass I) was originally proposed by the senior author in 1986, and details of the procedure were subsequently published in $1990^{2}$ (Fig. 1). This bypass is utilized for trapping intracavernous fusiform or giant aneurysms and carotid-cavernous fistulas, for bypassing cavernous CA stenoses, and for radical resection of vessel-engulfing tumors.

Following a standard frontotemporal-pterional craniotomy, the sphenoid ridge is drilled flat with a high-powered air drill. The epidural dissection is advanced to expose the frontal base and the anterior middle fossa. The extradural optic canal dura is exposed, and the anterior clinoid process is drilled away, detached from the optic strut, and removed. The superior orbital fissure, the foramen rotundum, and the foramen ovale are identified in the middle cranial fossa. The petrous segment of the CA is exposed by drilling the posterolateral Glasscock triangle medial to the foramen spinosum and the tensor tympani muscle. Drilling of the Glasscock triangle should be started just posterior to the foramen ovale and medial to the middle meningeal artery, thereby exposing the tensor tympani muscle and carefully skeletonizing the petrous CA. The greater superficial petrosal nerve is sacrificed with preservation of the geniculate ganglion. Facial nerve monitoring is utilized. At the proximal genu of the petrous segment of the CA, the cochlea is extremely close. Exposure of the petrous segment is facilitated by the skeletonization of the peripheral branch of the trigeminal third division and anterior translocation of the trigeminal ganglion.

The dura mater over the optic nerve is incised to expose the optic nerve widely and to dissect the ophthalmic artery. The optic nerve is gently retracted medially, and the fibrous ring of the paraclinoid junction is excised to mobilize the siphon segment of the CA. Bleeding from the cavernous

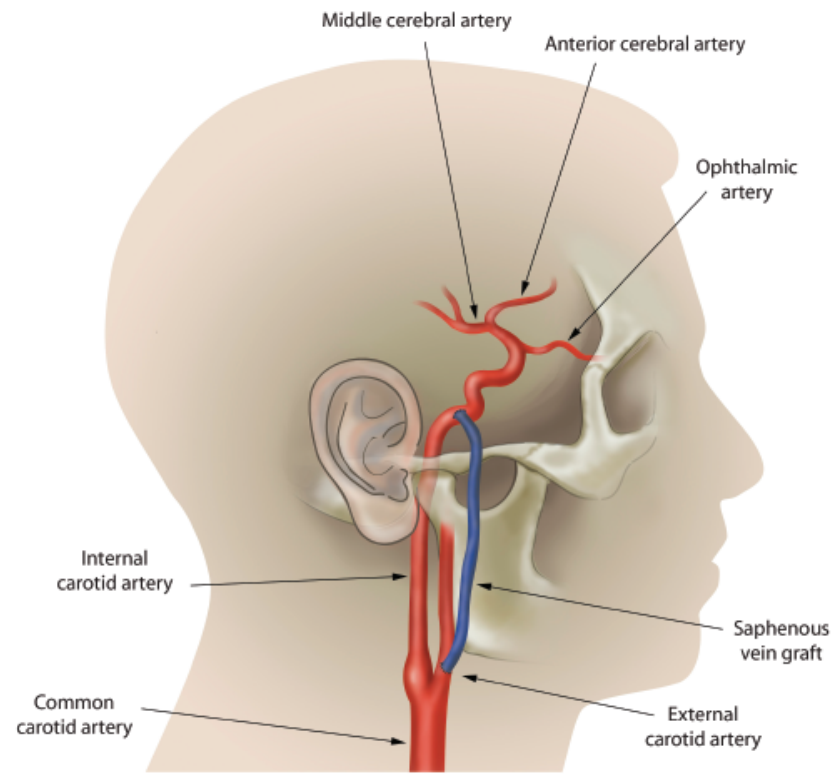

FIG. 2. Schematic illustration of Skull Base Bypass II.

sinus is controlled by packing with small pieces of Surgicel. In the earlier cases in which cavernous bypasses were performed, the frontal and temporal lobes were both retracted, sacrificing the temporal tip veins to make a direct saphenous vein interposition graft between the petrous segment of the CA and the siphon segment.

In the latter half of the bypass series, a keyhole extradural procedure was developed to preserve the temporal tip veins. In this extradural procedure, the dura mater of the temporal lobe is maintained, and the small epidural subtemporal approach is performed to expose the petrous CA segment. The saphenous vein is passed through a small dural incision from the subtemporal to the clinoidal area under the temporal lobe. The proximal CA is occluded by packing a small cottonoid into the carotid canal, and the CA is then ligated under the trigeminal ganglion at the petrous segment. The petrous segment of the CA is incised and an end-to-end anastomosis is performed with the saphenous vein, using 8-0 monofilament nylon interrupted sutures. At the distal anastomosis area, only the siphon segment of the ICA is exposed with a small dural incision, and the double clip is applied to the siphon to trap the aneurysm. The distal portion of the saphenous vein is anastomosed to the paraophthalmic area with an end-to-end anastomosis technique.

In most cases in our case series, the lengths of the saphenous vein grafts were 2-3 inches, and the grafts were harvested from the upper thigh. Before temporary occlusion of the CA, an intravenous bolus of 3000-5000 units of heparin was administered and the standard barbiturate cerebral protection procedure was undertaken. In the case of poor backflow from the distal $\mathrm{CA}$, a moderate hypothermia technique $\left(32-34^{\circ} \mathrm{C}\right)$ was utilized.

Before the last suture is completed, intraluminal blood or air is flushed away with heparinized saline. The drilled area is packed with pieces of pericranial and muscle graft or, occasionally, with abdominal fat grafting. 

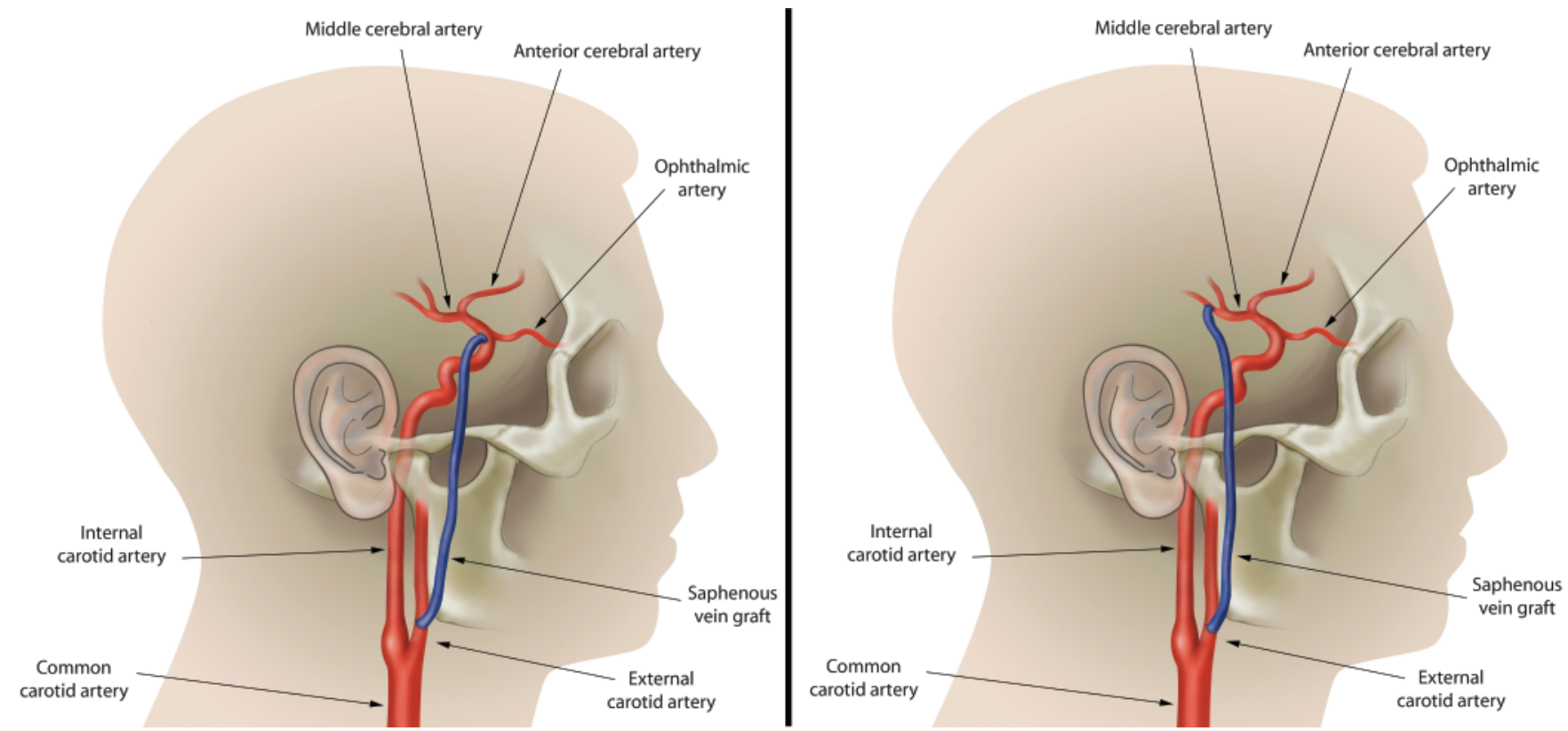

FIG. 3. Schematic illustrations of Skull Base Bypass III. The saphenous vein graft may be anastomosed to the siphon segment of the ICA (left) or to $\mathrm{M}_{2}$ (right).

The senior author predominantly utilized this procedure for cavernous CA aneurysms. The initial enthusiasm for the procedure was tempered by the risk of visual loss secondary to temporary clipping of the ophthalmic artery and the risk of facial nerve injury. Although this technique represents an excellent tool in the armamentarium of skull base and cerebrovascular neurosurgeons, we no longer use it widely. Of the 50 patients we treated for giant cavernous CA aneurysms, $4(8 \%)$ developed some degree of visual loss. In 1 patient, hemiplegia developed following graft thrombosis within a month of the procedure. Temporally this correlated with the patient stopping his antiplatelet regimen. It has been our practice to continue aspirin therapy indefinitely in patients who have undergone cerebral bypass surgery. No other graft occlusions were noted in either short- or long-term follow-up. In general, our practice has been to obtain a formal cerebral angiogram 6 months after the procedure, and then another at 1 year. If continued graft patency is noted, the patient is then followed up clinically. With the increased resolution of noninvasive screening methods such as CT angiography, we have now modified our practice, obtaining a formal angiogram at 6 months and, if there are no concerns regarding the graft, obtaining a CT angiogram 1 year postoperatively.

\section{Skull Base Bypass II}

The high-cervical external CA-petrous segment bypass (Skull Base Bypass II) is indicated for radical resection of high-cervical and infratemporal tumors encasing the ICA, repair of high-cervical CA aneurysms, and treatment of any occlusive ICA lesions in this segment (Fig. 2). Surgery is performed through a combined high-cervical incision and small extradural subtemporal exposure. In cases of neoplasia, the tumor is resected either through a high-cervical, tunnel operative approach or through a combined high-cervical extradural subtemporal exposure. After elimination of the lesion, a saphenous vein of appropriate length is anastomosed end to end to the ICA or end to side to the external CA in the neck, and then a disposable chest trocar tube is inserted from the neck through the submandibular pterygoid fossa up to the subtemporal epidural area. The other end of the saphenous vein is then inserted into the tube from the neck, and the vein is suctioned in the subtemporal direction through the tube. Once the vein is passed through the tube, the tube is removed. The distal end of the saphenous graft is connected extradurally to the petrous CA segment with an end-to-end anastomosis.

No short-term graft occlusions occurred in this case series. There was 1 long-term graft occlusion with no resultant deficits. In retrospectively reviewing this case, we noted that no technical problems were encountered at the time of surgery. Immediate angiography following the procedure revealed graft patency. It was unclear whether the patient had been compliant with antiplatelet therapy.

\section{Skull Base Bypass III}

The high-cervical segment-siphon (or $-\mathrm{M}_{2}$ ) saphenous vein graft (Skull Base Bypass III) bypass is indicated for cavernous sinus lesions that involve the petrous CA. In such cases, a saphenous vein graft is anastomosed from the high-cervical CA to the siphon (or $\mathrm{M}_{2}$; Fig. 3). Through a standard frontotemporal craniotomy, the proximal supraclinoid ICA is exposed by opening the anteromedial cavernous triangle and excising the fibrous carotid ring. Then the high-cervical CA is exposed by a routine neck procedure. The long saphenous vein graft is anastomosed first at the external CA and then past it, using a chest trocar technique through the submandibular pterygoid route to the 
K. R. Bulsara, T. Patel, and T. Fukushima

temporal base. The distal end of the saphenous vein is passed under the temporal lobe to the siphon area. No graft occlusions in the short-or long-term period were noted in this group of patients.

\section{Conclusions}

The ability to perform cerebral bypass surgery is an essential component in the armamentarium of skull base/cerebrovascular neurosurgeons. In this paper we summarize and describe our techniques and conceptually divide the bypasses into 3 groups.

\section{References}

1. Fukushima T, Sameshima T: Manual of Skull Base Dissection, ed 2. Pittsburgh: AF-Neurovideo, Inc, 2004

2. Spetzler RF, Fukushima T, Martin N, Zabramski JM: Petrous carotid-to-intradural carotid saphenous vein graft for intracavernous giant aneurysm, tumor, and occlusive cerebrovascular disease. J Neurosurg 73:496-501, 1990

Manuscript submitted November 15, 2007.

Accepted December 10, 2007.

Address correspondence to: Takanori Fukushima M.D., D.S.M., Carolina Neuroscience Institute, 4030 Wake Forest Road, Suite 115, Raleigh, North Carolina 27609. email: fukushima@ carolinaneuroscience.com. 